\title{
The association between Situational Judgement Test (SJT) scores and Professionalism Concerns in Undergraduate Medical Education Sahota GS ${ }^{1}$, Taggar JS
}

${ }^{1}$ Primary Care Education Unit, Division of Primary Care, University of Nottingham, UK

${ }^{2}$ Medical Education Centre, University of Nottingham, UK

Running head: SJT associations with student professionalism

Corresponding author:

Gurvinder Sahota

Room C38, C floor

Division of Primary Care

University of Nottingham

NG7 2UH

Tel: +44 (0)1158230462

Email: Gurvinder.sahota@nottingham.ac.uk

Keywords: Situational Judgement Test, SJT, Professionalism assessment, Professionalism concerns

$\underline{\text { Notes on contributors }}$

Dr Gurvinder Sahota is a clinical academic and educator, responsible for first and second year medical students' clinical and professional development at Nottingham Medical School.

Dr Jaspal Taggar is a Clinical Associate Professor and educator, who is Head of the Primary Care Education Unit at Nottingham University. He is responsible for strategic oversite and quality assurance of clinical placements and undergraduate primary care education at Nottingham Medical School.

Word Count: 4003 words (Abstract: 194. Article: 3,474, Tables: 235) 


\section{Abstract \\ Introduction}

Situational judgement tests (SJTs) are widely used in selecting medical students and doctors. Emerging evidence suggests SJTs are capable of testing an individual's ability to respond to role-relevant professionalism scenarios, however evidence is lacking for their use in identifying students with concerning professional behaviours.

This study aimed to determine the association between medical student professionalismbased SJT scores and recorded professionalism concerns during training.

\section{Methods}

A cross-sectional study was conducted utilising SJT scores from second-year medical students and occurrences of student professionalism concerns. Concerns were reviewed and mapped to General Medical Council standards. Multivariate logistic regression was used to determine associations between SJT scores and professionalism concerns.

\section{Results}

247 students were included in the study. For every point increase in SJT score, students were $10 \%$ less likely to have multiple professionalism concerns [OR(95\%CI)0.90(0.83$0.97) ; \mathrm{p}=0.007]$.

Students scoring below 1 and 2 standard deviations from the mean score were 4 and 11times more likely to have multiple concerns [OR(95\%CI)4.52(1.12-18.25); $\mathrm{p}=0.034]$ \& [OR(95\%CI)11.45(1.72-76.15); $\mathrm{p}=0.012]$.

\section{Conclusion}

Lower SJT scores were significantly associated with an increased risk of professionalism concerns. These findings support the potential for SJT exams to identify medical students that may require closer supervision and remediation during undergraduate education.

(194 words) 


\section{Introduction}

Professionalism has been recognised as an essential attribute to medical practice for thousands of years. Identifying students with unprofessional behaviours during medical school has become an important area of research, as postgraduate professionalism breaches have been associated with concerns identified during undergraduate training (Papadakis et al. 2004). Unprofessional behaviours are associated with reduced patient satisfaction (Bahaziq and Crosby 2011) whilst good professionalism can improve medical outcomes (Brennan and Monson 2014) and be associated with excellence in medical knowledge, skills and attitudes (Reed et al. 2008). Assessment of professionalism is, therefore, of paramount importance to medical schools and regulators internationally but there is no definitive assessment method for it (Medical Professionalism Project 2002; Royal College of Physicians 2005; O'Sullivan et al. 2012; General Medical Council 2013; Accreditation Council for Graduate Medical Education 2017; General Medical Council 2018).

Situational judgement tests (SJTs) are a relatively new assessment modality for professionalism. Research suggests they have good reliability and psychometric robustness in testing non-academic attributes of medical students (Husbands et al. 2015; Patterson et al. 2016; Goss et al. 2017). SJT questions are designed to test an individual's ability to respond to scenarios in a plausible, role-relevant situation. Moreover, assessment of professionalism has been advocated during early medical training to promote learning and professional identity formation (Boon and Turner 2004; Kalet et al. 2017; Houtlosser et al. 2018).

SJTs often test procedural, ethical and legal constructs and have demonstrated associations with existing assessment metrics, such as postgraduate educational supervisor ratings, clinical examination scores and medical simulation testing (Patterson et al. 2009; Lievens and Patterson 2011; Koczwara et al. 2012). Additionally, they may predict scoring of professional attributes such as communication and integrity (Patterson et al. 2017). SJTs focussing on aspects of professionalism have a history of being utilised as an assessment tool within dental and medical schools (Schubert et al. 2008; Ashworth et al. 2015; Husbands et al. 2015; Goss et al. 2017). Indeed, they are one of a select few tools that the General Medical Council (GMC) recommend for assessing undergraduate medical professionalism (General Medical Council 2011). 
Whilst there is emerging evidence about the utility of SJTs as an assessment tool for medical professionalism (Goss et al. 2017), there is little evidence for their relationship with observed behaviours and breaches of professionalism during medical training. It is crucial that medical schools not only have tools that enable the selection of appropriate students to courses, but also assessments that enable the identification of students that exhibit concerning behaviours that require remediation. Although identified as an area needing exploration (Goss et al. 2017), there is no research that assesses the direct association between SJT scores and observed professionalism concerns in undergraduates.

Therefore, the aims of this study were to determine the association between student SJT scores and recorded professionalism concerns. 


\section{Methods}

\section{Setting and participants}

The study was conducted at the University of Nottingham, United Kingdom (UK), where there has been a mandatory professionalism-based SJT summative assessment integral to the professionalism curriculum since 2014 (Sahota et al. 2014). The SJT examines students at the end of their second year of study. The first two years of the Nottingham medical course is comprised of university-based teaching of biomedical sciences and professionalism. Professionalism teaching occurs via lectures, seminars (on bioethics; communication; medico-legal issues) and observational clinical visits in primary and secondary care. From the third year onwards the course transitions mainly to a clinical placements setting.

The study cohort included all undergraduate students that sat the professionalism-based SJT in May 2014.

\section{Data collection}

\section{Participant characteristics}

Participant gender was ascertained as research suggests gender may be a confounder in the association between SJT scores and professionalism concerns (Whetzel et al. 2008; Lievens 2013; Yates 2014; Harris 2015; Luschin-Ebengreuth et al. 2015; Jardine et al. 2017).

\section{Explanatory variables: Situational Judgement Tests (SJTs) scores}

Example SJT questions used in the assessment are provided in appendix 1. A professionalism-based SJT assesment was derviced using the followng process to ensure face and construct validity of the SJT. Questions were derived through consensus by a panel of six subject matter experts at the University of Nottingham. Experts were members of the early clinical and professional development academic team and utilised real life critical incidents as a basis for SJT scenarios. Questions were formulated using the standards set out in the GMC's Good Medical Practice document (General Medical Council 2013), which forms the basis of the professionalism teaching that students receive during the medical course at Nottingham. This question development process was 
in accordance to the Association for Medical Education in Europe best practice guidance (Patterson et al. 2016).

Questions were abandoned if there was ambiguity in construct or unresolvable differences in deciding the answer (Patterson et al. 2016). Questions approved by the panel were sent to an external examiner (independent of question development) to check for relevance and fairness, in accordance with the Nottingham School of Medicine governance processes.

Question style for the SJT was selected as ranking 'best of five responses' and 'best three reponses' as these align with the national SJT selection process for junior doctors in the United Kingdom (United Kingdom Foundation Programme Office 2018).

The exam comprised 27 SJT questions - 12 ranking 'best of five responses' and 9 'best three responses' questions (appendix 1) - with a total of 201 attributed marks. Students were examined using the Rogo software - a secure, computer based method for electronic examinations. Students were allowed 90 minutes to complete the assessment under strict examination conditions.

In keeping with SJT marking systems, ranking questions were marked by a partial credit system (United Kingdom Clinical Aptitude Test 2016). Students could score a maximum of 10 marks for ranking 'best of five responses' questions and a maximum of 9 marks for 'best three responses' questions. This marking system still allowed a possible score of zero.

For the purpose of analyses, SJT scores were used as i) a continuous variable ii) dichotomised as scores less than 1 standard deviation below the mean SJT score and scores above this, and iii) dichotomised as scores less than 2 standard deviations below the mean SJT score and scores above this.

Dichtomising the SJT scores using the cut-points described above aligned with the accepted convention of norm-referencing SJT scores in the national UK Foundation Programme examination (McLachlan and Illing 2013).

In keeping with SJT evidence, reliability of the study's SJT was measured using Cronbach's alpha (Colbert-Getz et al. 2015; Kasten and Freund 2016; Patterson et al. 2016; Goss et al. 2017). No accepted alpha scores exist specifically for SJTs and recent undergraduate professionalism studies have quoted alphas as between 0.45-0.64 
(Husbands et al. 2015; Goss et al. 2017). Cronbach's alpha of the SJT for this study was 0.524 .

\section{Outcome variables: Student professionalism concerns}

The outcome measure for this study was the number of occurences of formal professionalism concerns about medical students. Notification and management of concerns in professionalism using a 'concern form' tool is a recognised and advocated approach by institutions and medical regulators internationally (Yates 2014; Ziring et al. 2015; General Medical Council and Medical Schools Council 2016; College of Medicine 2017; University of Toronto 2019).

At Nottingham Medical School professionalism concerns can be formally raised by staff and/or students using a concern form. These forms are widely available and electronically submitted to the central medical school team. Concerns raised are collated and recorded on a central electronic database. For this study, data for concerns raised about student professionalism were reviewed in January 2018. Concern forms included in the analyses were from the duration of study cohorts medical training (i.e. years 1-5 of the BMBS degree; 2012- 2017).

Submitted concern forms were identified via a search of the electronic university database and linked, using student identification numbers, to students that sat the exam and had recorded SJT scores. All concern forms were manually checked twice to ensure they constituted a breach of standards within the GMCs Good Medical Practice document (General Medical Council 2013). Concerns were then categorised into the four Good Medical Practice domains:

- Domain 1: Knowledge, skills and performance

- Domain 2: Safety and quality

- Domain 3: Communication, partnership and teamwork

- Domain 4: Maintaining trust.

Forms relating to student mental health were not included in the analyses as recommended in previous research (Papadakis et al. 2004). 
Student outcomes were categorised into the following dichotomised groups: i) any professionalism concern and no professionalism concerns ii) one professionalism concern and no professionalism concerns and iii) more than one professionalism concern and no professionalism concerns.

\section{Statistical analysis}

Analyses were conducted using Statistical Package for the Social Sciences (IBM SPSS Statistics for Windows, Version 24.0. Armonk, NY: IBM Corp.). Categorical data were summarised using numbers and percentages. Continuous data were summarised using means and standard deviations (SDs).

Associations between SJT scores and professionalism concerns were determined using multivariate logistic regression. Multivariate logistic regression enables derviation of statistical models to determine associations between variables whilst adjusting for the effects of potential confounding factors. The strength of assocations are expressed as odds ratios and the statistical significance of associations are expressed using $\mathrm{P}$ values.

In this study regression models were used to determine whether lower SJT scores were associated with an increased likelihood of students having professionalism cocerns. Gender was included as an independent variable in the regression models as previous research suggests male students are more likely to score lower in SJTs and receive greater professionalism concerns. Adjusting for confounding allowed for greater precision precision within the analyses (Whetzel et al. 2008; Lievens 2013; Yates 2014; Harris 2015; Luschin-Ebengreuth et al. 2015; Jardine et al. 2017).

The SJT variables were entered into models as both continuous and categorised variables, the latter using cut-points as described previously. Students with scores below the cutpoints (i.e. lower SJT scores) were compared to those with scores above the cut-points (i.e. higher SJT scores). Professionalism concerns were entered into analyses as dichotomous variables as previously described. A $\mathrm{P}<0.05$ was used to indicate a statistically significant association.

\section{Ethical considerations}

Ethical approval was granted for this study by the University of Nottingham's Research Ethics Committee. 


\section{Results}

\section{Participant characteristics}

There were 247 students included in the study of which $151(61.1 \%)$ were female. The data for numbers of students within categories of SJT scores and professionalism concerns used within analyses are provided in table 1.

[Table 1 near here]

\section{Situational Judgement Test (SJT) Scores}

A total of 201 marks were available in the SJT. Scores ranged from 121 (minimum) to 174 (maximum) marks. The mean SJT score was 151.1 (8.9). The threshold for mean minus 1 SD for SJT score was 142.2 marks; 45 (18.2\%) students scored below this threshold. The threshold for mean minus 2 SDs for SJT score was 133.3 marks; 7 (2.8\%) students scored below this threshold.

\section{Professionalism concerns}

Of the 247 students, $24(9.7 \%)$ had recorded professionalism concerns. There were 9 (3.6\%) students that had more than one professionalism concern.

In total there were 37 forms submitted for the 24 students with professionalism concerns. Of the four GMC professionalism categories, $49 \%$ were categorised into Domain 1 (Knowledge, skills and performance); $43 \%$ into Domain 3 (communication, partnership and teamwork); and 8\% for Domain 4 (maintaining trust). There were no concerns that identified breaches within Domain 2 (Safety and quality). There were no forms where there was a breach of more than one professionalism domain.

Examples of concerns raised and the domains within which they were categorised include:

Domain 1: poor attendance at university or clinical teaching sessions

Domain 3: lack of response to faculty communications; disrespectful communication to staff

Domain 4: fabrication of reasons for absences; halls of residence vandalism. 


\section{Association between Situational Judgement Test scores and professionalism concerns}

The multivariate analyses for associations between SJT scores and professionalism concerns are provided in table 2.

There was a borderline significant association between increasing SJT score as a continuous variable and students having any number of professionalism concerns; for every one point increase in SJT score there was a 5\% lower risk of professionalism concerns [OR (95\% CI) $0.95(0.91-1.00) ; \mathrm{p}=0.049]$. The strength and significance of this association was greater when SJT scores were analysed in students with more than one professionalism concern. Each point increase in SJT score was associated with a $10 \%$ lower risk of multiple professionalism concerns [OR (95\% CI) 0.90 (0.83-0.97); $\mathrm{p}=0.007]$.

When SJT scores were entered into the analysis as categoried variables, there were statistically significant inverse associations between SJT scores and students with multiple professionalism concerns. Using a cut-point of 1 SD below the mean SJT score, those scoring below this cut-point were 4-times more likely to have multiple professionalism concerns than those scoring above this [OR (95\% CI) 4.52 (1.12-18.25); $\mathrm{p}=0.034]$. Using a cut-point of 2 SDs below the mean SJT score, students scoring below this cut-point were 11-times more likely to have multiple professionalism concerns than those scoring above this [OR $(95 \% \mathrm{CI}) 11.45(1.72-76.15) ; \mathrm{p}=0.012]$.

There were no significant associations between SJTs scores and professionalism concerns in students where only one concern was identified.

[Table 2 near here] 


\section{Discussion}

This study found a significant inverse association between SJT scores and observed multiple professionalism concerns in medical students. For each point increase in SJT score, students were $10 \%$ less likely to have multiple professionalism concerns. Students who scored below one and two standard deviations from the mean SJT score were 4 and 11 times more likely to have multiple professionalism concerns than their peers with higher SJT scores, respectively.

As recommended by regulators internationally, medical schools must ensure students graduating as doctors have professionalism as a core competency. This study demonstrates that professionalism-based SJTs may help identify students at greatest risk of exhibiting multiple unprofessional behaviours during training. This could be used to target interventions, through support and remediation, that enable further professional development. Ultimately, this would strenghten confidence of educators and regulators that the 'most professional' students are graduating from institutions.

Given the novelty of this study, there is a paucity of literature for direct comparison of the findings. Existing evidence suggests medical school professionalism concerns may be associated with unprofessional behaviours in postgraduate practice (Papadakis et al. 2004) and the medical school clinical years (Burns et al. 2017). We believe this is the first study to determine an association between summative professionalism-based SJT scores and formally documented professionalism concerns, representating unprofessional behaviours, in undergraduate medical education.

Consistent with existing literature, this study demonstrated the majority of students in the cohort had no professionalism concerns identified, with less than 10 percent having any professionalism concern and less than four percent having multiple concerns. (McLachlan et al. 2009; Yates 2014; Ainsworth and Szauter 2018).

Conversely, our study identified nearly twice as many concerns than the conscientious index (CI) study, which also utilised the GMC's Good Medical Practice as its basis for professional standards (McLachlan et al. 2009). McLachlan et al. attempted to analyse the relationship between medical student's critical incident forms and their CI but were unable due to insufficient sample size. However, they inferred a "relationship does seem to exist but is not yet quantifiable" as around $70 \%$ of critical incident forms were from students with low CI scores (McLachlan et al. 2009 p. 563). 
There is little published evidence for SJT threshold scores that could be used to identify unprofessional or lower professional students. Evidence indicates that SJTs used in selection may have better predictive validity for lower performers and they could be used to 'select out' poorer students (Patterson et al. 2016 p. 9). The literature is not clear on how to identify a mark and there is no specific cut score or accepted standard setting method, which poses great difficulty for educators attempting to use this tool. SJTs used in selection often use a norm-referencing standard deviation approach, with the UK foundation application SJT quoting thresholds of 2.5 or 4 standard deviations away from the mean as a cut score for candidates (McLachlan and Illing 2013). In contrast, our study found significant associations for multiple professionalism concerns in students scoring below one standard deviation from the mean. Moreover the strength and significance of this association increased when the cut-point of SJT scores was varied from one to two standard deviations below the mean. This has important practical implications - firstly it strengthens the validity of findings by suggesting a 'dose-response effect' and, secondly, for medical educators it provides an indication to the cut-points that could be used in professionalism-based SJT exams for identifying students that may need remediation.

Furthermore, this study's findings demonstrate that SJT scores were not associated with students only receiving one concern. This supports the notion 'once is a mistake and twice is a choice' and highlights the SJTs ability to better identify those at greatest risk of sustained unprofessional behaviours.

The use of a professionalism SJT or indeed any explicit method of professionalism teaching or assessment demonstrates to students that specific attributes of professional behaviour need to be exhibited in order for students to progress in their education. From a student perspective, assessment drives learning (Wormald et al. 2009; Schuwirth and Van Der Vleuten 2011) and the use of the SJT examination may have influenced students to behave more professionally, thus reducing subsequent breaches in professionalism - a phenomenon reported by Goss et al (Goss et al. 2017). This has the potential to have reduced the strength of associations reported in our study. 


\section{Strengths and limitations}

To our knowledge this is the first study that has determined the direct association between medical student SJT scores and concerns in professionalism.

The study collected data from a single institution which limits the generalisability of findings. Other institutes may have different approaches to assessing and/or managing professionalism concerns which could influence the results in either direction. Therefore, replication of the findings in other medical institutes would strengthen the findings. This study looked at a single cohort of students with a relatively low sample size $(n=247)$ as reflected by the wide confidence intervals for reported associations. Whilst all student data were utilised, thus minimising potential selection bias, the small sample size could result in the potential for chance findings.

Concern form information was collected by numerous individuals at various stages of students' training. This has multiple implications; firstly staff and/or students must recognise professionalism concerns of sufficient severity requiring the formal submission of a form. Secondly, other mechanisms such as private meetings between staff and students may have taken place without a form being submitted. It is therefore possible that the recorded professionalism concerns may under represent the frequency of real-life concerns. This makes it more likely that the concerns raised were, in fact, of sufficient severity thus improving the robustness of outcomes in the study. To reduce the potential for misclassification bias, concern forms in this study were reviewed and mapped against GMC Good Medical Practice standards further improving the validity of the findings.

Cronbach's alpha is a measure of internal consistency of assessments. It could be argued that the Cronbach's alpha for the SJT in this study was low at 0.525 , thus limiting the reliability of the exam. Suggested ideal values are 0.7 to 0.9 and asssessments with values over 0.9 are likely to include redundant questions (Bland and Altman 1997; Streiner 2003; Tavakol and Dennick 2011). However, no accepted alpha scores exist specifically for SJTs and this study's alpha was similar to other studies investigating undergraduate professionalism; acceptable alphas in such cases have been between 0.45- 0.64 (Husbands et al. 2015; Goss et al. 2017).

Finally, this study utilised robust statistical analyses to determine the association between SJT scores and professionalism concerns and precision of findings was improved by 
adjusting for confounding by gender. However, the potential for residual confounding remains a possibility.

\section{Conclusion}

This study found a significant inverse association between SJT scores and the risk of multiple professionalism concerns. Students who scored lower than 2 standard deviations from the mean SJT score were 11 times more likely to have multiple professionalism concerns than their peers with SJT scores above this.

Further research that tests the generalisability, causality of association and predictive validity of SJT scores for unprofessional behaviours in undergraduate medical education is required.

\section{Practical implications}

This study supports the use of professionalism focussed SJTs as an undergraduate medical school assessment. It suggests that SJT scores of 1 and 2 standard deviations below the mean SJT score could enable the identification of students with unprofessional behaviours who could benefit from closer supervision and/or remediation.

\section{Practice points}

- Situational judgement tests can be used as a medical student professionalism assessment

- Lower scoring students are more likely to have professionalism concerns than their peers with higher SJT scores

- A cut score of 2 standard deviations below a mean SJT score could be used to identify students for remediation or closer supervision 


\section{$\underline{\text { Acknowledgments }}$}

Thanks to Dr Edward Tyrrell for assistance in statistical analysis of the datasets.

$\underline{\text { Declaration of Interest }}$

The authors report no declarations of interest. 


\section{$\underline{\text { References }}$}

1. Accreditation Council for Graduate Medical Education. 2017. Accreditation Council for Graduate Medical Education: Program Requirements for Graduate Medical Education in Pediatrics.

https://www.acgme.org/Portals/O/PFAssets/ProgramRequirements/320 pediatrics 20

17-07-01.pdf. [Accessed 18 January 2019].

2. Ainsworth MA, Szauter KM. 2018. Student response to reports of unprofessional behavior: assessing risk of subsequent professional problems in medical school. Medical education online. 23(1):1485432-1485432.

3. Ashworth V, Escudier M, Flaxman C, Cousans F, Woolford M, Patterson F. 2015. Pilot results from a new assessment of professionalism. AMEE Conference; Glasgow, UK.

4. Bahaziq W, Crosby E. 2011. Physician professional behaviour affects outcomes: a framework for teaching professionalism during anesthesia residency. Canadian journal of anaesthesia $=$ Journal canadien d'anesthesie. 58(11):1039-1050. eng.

5. Bland JM, Altman DG. 1997. Statistics notes: Cronbachs alpha. BMJ (Clinical research ed). $314(7080): 572$.

6. Boon K, Turner J. 2004. Ethical and professional conduct of medical students: review of current assessment measures and controversies. Journal of Medical Ethics. 30(2):221226.

7. Brennan MD, Monson V. 2014. Professionalism: good for patients and health care organizations. Mayo Clinic proceedings. 89(5):644-652. eng.

8. Burns CA, Lambros MA, Atkinson HH, Russell G, Fitch MT. 2017. Preclinical medical student observations associated with later professionalism concerns. Medical teacher. 39(1):38-43.

9. Colbert-Getz JM, Pippitt K, Chan B. 2015. Developing a situational judgment test blueprint for assessing the non-cognitive skills of applicants to the University of Utah 
School of Medicine, the United States. Journal of educational evaluation for health professions. 12:51. eng.

10. College of Medicine. 2017. Procedures for Concerns with Medical Student Professional Behaviour. Procedures for Concerns with Medical Student Professional Behaviour: University of Saskatchewan College of Medicine; [accessed 201909 January]. https://medicine.usask.ca/policies/professionalism-standard-operatingprocedure.php\#1Purpose

11. General Medical Council. 2011. Assessment in undergraduate medical education. https://www.gmc-uk.org/Lmedia/documents/Assessment in undergraduate medical education guidance 0 815.pdf 56439668.pdf: General Medical Council; [accessed 2019 7th January].

12. General Medical Council. 2013. General Medical Council: Good Medical Practice. Avalable from: https://www.gmcuk.org/static/documents/content/Good medical practice - English 1215.pdf; [accessed 12/11/17].

13. General Medical Council. 2018. General Medical Council: Outcomes for Graduates. Available from: https://www.gmc-uk.org/-/media/documents/dc11326-outcomes-forgraduates-2018 pdf-75040796.pdf; [accessed 30/07/18].

14. General Medical Council, Medical Schools Council. 2016. General Medical Council and Medical School Council. Professional behaviour and fitness to practise: guidance for medical schools and their students. Available from:https://www.gmc-uk.org/Lmedia/documents/Professional behaviour and fitness to practise 0816.pdf 66085 925.pdf; [accessed 03/03/18].

15. Goss BD, Ryan AT, Waring J, Judd T, Chiavaroli NG, O'Brien RC, Trumble SC, McColl GJ. 2017. Beyond Selection: The Use of Situational Judgement Tests in the Teaching and 
Assessment of Professionalism. Academic medicine : journal of the Association of American Medical Colleges. 92(6):780-784.

16. Harris R. 2015. Analysis of cases resulting in doctors being suspended or erased from the medical register. https://www.gmc-uk.org/-

Lmedia/documents/Analysis of cases resulting in doctors being suspended or era sed from the medical register FINAL REPORT Oct 2015.pdf 63534317.pdf. Accessed 18 December 2018: DJS research report on behalf of General Medical Council.

17. Houtlosser M, Ottenhoff-de Jonge MW, Essers GTJM, Numans ME, Kramer AWM. 2018. A practical framework for remediating unprofessional behavior and for developing professionalism competencies and a professional identity AU - Barnhoorn, Pieter C. Medical Teacher.1-6.

18. Husbands A, Rodgerson MJ, Dowell J, Patterson F. 2015. Evaluating the validity of an integrity-based situational judgement test for medical school admissions. Bmc Medical Education. 15.

19. Jardine DL, McKenzie JM, Wilkinson TJ. 2017. Predicting medical students who will have difficulty during their clinical training. BMC Med Educ. 17(1):43. eng.

20. Kalet $A$, Chou CL, Ellaway RH. 2017. To fail is human: remediating remediation in medical education. Perspectives on medical education. 6(6):418-424.

21. Kasten N, Freund PA. 2016. A Meta-Analytical Multilevel Reliability Generalization of Situational Judgment Tests (SJTs). European Journal of Psychological Assessment. $32(3): 230-240$.

22. Koczwara A, Patterson F, Zibarras L, Kerrin M, Irish B, Wilkinson M. 2012. Evaluating cognitive ability, knowledge tests and situational judgement tests for postgraduate selection. Medical education. 46(4):399-408. 
23. Lievens F. 2013. Adjusting medical school admission: assessing interpersonal skills using situational judgement tests. Medical education. 47(2):182-189.

24. Lievens F, Patterson F. 2011. The Validity and Incremental Validity of Knowledge Tests, Low-Fidelity Simulations, and High-Fidelity Simulations for Predicting Job Performance in Advanced-Level High-Stakes Selection. Journal of Applied Psychology. 96(5):927-940.

25. Luschin-Ebengreuth M, Dimai HP, Ithaler D, Neges HM, Reibnegger G. 2015. Situational judgment test as an additional tool in a medical admission test: an observational investigation. BMC Research Notes. 8.

26. McLachlan J, Finn G, Macnaughton J. 2009. The conscientiousness index: A novel tool to explore students' professionalism. Academic Medicine. 84(5):559-565.

27. McLachlan J, Illing J. 2013. Health Education England report: An independent review of events relating to the use of situational judgement tests in the selection for foundation process 2013. Available from: https://www.medschools.ac.uk/media/1932/heeindependent-review-of-the-sjt.pdf; [accessed 18/01/18].

28. Medical Professionalism Project. 2002. Medical Professionalism Project (MPP) Medical professionalism in the new millennium: a physician charter. Ann Intern Med. 136(3):243-246. eng.

29. O'Sullivan H, van Mook W, Fewtrell R, Wass V. 2012. Integrating professionalism into the curriculum: AMEE Guide No. 61. Medical teacher. 34(2):e64-77. eng.

30. Papadakis MA, Hodgson CS, Teherani A, Kohatsu ND. 2004. Unprofessional behavior in medical school is associated with subsequent disciplinary action by a state medical board. Academic medicine : journal of the Association of American Medical Colleges. 79(3):244-249. eng.

31. Patterson F, Baron H, Carr V, Plint S, Lane P. 2009. Evaluation of three short-listing methodologies for selection into postgraduate training in general practice. Medical Education. 43(1):50-57. 
32. Patterson F, Lopes S, Harding S, Vaux E, Berkin L, Black D. 2017. The predictive validity of a situational judgement test, a clinical problem solving test and the core medical training selection methods for performance in specialty training. Clinical medicine (London, England). 17(1):13-17. eng.

33. Patterson F, Zibarras L, Ashworth V. 2016. Situational judgement tests in medical education and training: Research, theory and practice: AMEE Guide No. 100. Medical Teacher. 38(1):3-17.

34. Reed DA, West CP, Mueller PS, Ficalora RD, Engstler GJ, Beckman TJ. 2008. Behaviors of highly professional resident physicians. Jama. 300(11):1326-1333. eng.

35. Royal College of Physicians. 2005. Royal College of Physicians Working Party Report. Doctors in Society: Medical professionalism in a changing world. . Available from: https://cdn.shopify.com/s/files/1/0924/4392/files/doctors in society reportweb.pdf? 15745311214883953343; [accessed 12/10/17].

36. Sahota G, Lee S, Knox R, Charlton R. 2014. Review of undergraduate medical use of situational judgement tests. The Association for the study of Medical Education Annual Scientific Meeting; Brighton.

37. Schubert S, Ortwein H, Dumitsch A, Schwantes U, Wilhelm O, Kiessling C. 2008. A situational judgement test of professional behaviour: development and validation. Medical teacher. 30(5):528-533. eng.

38. Schuwirth LWT, Van Der Vleuten CPM. 2011. Programmatic assessment: From assessment of learning to assessment for learning. Medical Teacher, 2011, Vol33(6), p478-485. 33(6):478-485.

39. Streiner DL. 2003. Starting at the beginning: an introduction to coefficient alpha and internal consistency. J Pers Assess. 80(1):99-103. eng.

40. Tavakol M, Dennick R. 2011. Making sense of Cronbach's alpha. 2:53. 
41. United Kingdom Clinical Aptitude Test. 2016. United Kingdom Clinical Aptitude Test: Technical Report. Available from: https://www.ukcat.ac.uk/media/1057/ukcat-2016technical-report-exec-summary v1.pdf; [accessed 10/03/18].

42. United Kingdom Foundation Programme Office. 2018. United Kingdom Foundation Programme Office. Situational Judgement Test (SJT): How do applicants perform in the SJT? Available from: http://www.foundationprogramme.nhs.uk/content/situationaljudgement-test-sit; [accessed 18/06/18].

43. University of Toronto. 2019. Professionalism. Univeristy of Toronto Faculty of Medicine; [accessed 20198 January]. http://ttrfd.med.utoronto.ca/professionalism.

44. Whetzel DL, McDaniel MA, Nguyen NT. 2008. Subgroup Differences in Situational Judgment Test Performance: A Meta-Analysis. Human Performance. 21(3):291-309.

45. Wormald BW, Schoeman S, Somasunderam A, Penn M. 2009. Assessment Drives Learning: An Unavoidable Truth? Anatomical Sciences Education. 2(5):199-204.

46. Yates J. 2014. "Concerns" about medical students' adverse behaviour and attitude: an audit of practice at Nottingham, with mapping to GMC guidance. BMC medical education. 14:196-196.

47. Ziring D, Danoff D, Grosseman S, Langer D, Esposito A, Jan MK, Rosenzweig S, Novack D. 2015. How Do Medical Schools Identify and Remediate Professionalism Lapses in Medical Students? A Study of U.S. and Canadian Medical Schools. Academic medicine : journal of the Association of American Medical Colleges. 90(7):913-920. eng. 


\section{$\underline{\text { Tables }}$}

Table 1: Summary of the characteristics of the study cohort

\begin{tabular}{|l|l|}
\hline Characteristic $(\mathrm{n}=247)$ & $\mathrm{n}(\%)$ \\
\hline SJT Score (1 SD below mean) & \\
\hline Below cut point (151 marks or below) & $45(18.2)$ \\
\hline Above cut point (152 marks or below) & $202(81.8)$ \\
\hline SJT Score (2 SD below mean) & \\
\hline Below cut point (142 marks or below) & $7(2.8)$ \\
\hline Above cut point (143 marks or below) & $240(97.2)$ \\
\hline Professionalism concerns & \\
\hline No concerns & $223(90.3)$ \\
\hline Any concern & $24(9.7)$ \\
\hline 1 concern & $15(6.1)$ \\
\hline$>1$ concern & $9(3.6)$ \\
\hline Professional concern GMC domains & \\
\hline 1: Knowledge, skills and performance & $18(48.6)$ \\
\hline 2:Safety and quality & $0(0)$ \\
\hline 3: Communication, partnership and teamwork & $16(43.2)$ \\
\hline 4: Maintaining trust & $3(8.1)$ \\
\hline
\end{tabular}


Table 2: Multivariate associations between SJT scores and professionalism concerns

\begin{tabular}{|c|c|c|c|c|c|c|}
\hline \multirow{3}{*}{ SJT scores } & \multicolumn{6}{|c|}{ Outcomes** } \\
\hline & \multicolumn{2}{|c|}{ Any concern } & \multicolumn{2}{|c|}{1 concern } & \multicolumn{2}{|l|}{$>1$ concern } \\
\hline & OR $(95 \% \mathrm{CI})$ & $P$ value & OR (95\% CI) & $P$ value & OR $(95 \% \mathrm{CI})$ & $P$ value \\
\hline SJT (continuous) & $0.95(0.91-1.00)$ & 0.049 & $0.99(0.93-1.05)$ & 0.791 & $0.900(0.834-0.972)$ & 0.007 \\
\hline SJT (1SD)* & $2.10(0.82-5.41)$ & 0.125 & $1.04(0.27-3.95)$ & 0.957 & $4.52(1.12-18.25)$ & 0.034 \\
\hline SJT (2SD)* & $7.07(1.44-34.78)$ & 0.016 & $2.54(0.28-22.84)$ & 0.405 & $11.45(1.72-76.15)$ & 0.012 \\
\hline
\end{tabular}

$\mathrm{SJT}=$ Situational Judgement Test; $\mathrm{SD}=$ Standard Deviation; $\mathrm{OR}=$ odds ratio; $\mathrm{CI}=$ confidence interval

*students with scores below 1 or 2 standard deviations from the mean SJT score (lower SJT scores) were compared to students with scores above these cut-points (SJT scores); below 1 SD is 151 marks or below and 2 SD is 142 marks or below; **concerns compared to no concerns 


\section{Appendices}

\section{Appendix 1.}

Example situational judgement questions

Questions 1 and 2 are example questions from the study SJT. Further questions from the actual examination are not reproduced within the dissertation as they are still actively used in summative examinations in the University of Nottingham's medical course.

Question 1 ranking and Questions 2 is choose bets of three style.

1.

You are a medical student shadowing a junior doctor. You are observing him taking consent for an operation from $\mathrm{Mr} \mathrm{R}$, a patient on the ward.

It seemed clear to you yesterday that Mr R did not understand English well and an interpreter's services were necessary in order to take a history from him. At that time he had expressed that he did not want an operation.

The doctor explains the procedure in English, with the patient nodding, Mr R signs the consent form and it is filed in his notes.

Rank in order the following actions in response to this situation (1= Most appropriate; $5=$ least appropriate)
A. Call the on-call consultant to inform him what has just happened
B. Inform the doctor that you think he needs an interpreter for the consent process
C. Go onto the internet and get a translation asking questions about the procedure and ascertain the patient's wishes
D. Carry on observing, as the patient can always say no when taken to theatre
E. Put a cross through the consent form in the notes invalidating it

Subject matter expert decided answer key: BAECD

Second example question from study SJT. 


\section{2.}

You are a junior doctor working at a weekend. You have called your consultant to review a sick patient, who is to be taken straight to the operating theatre. Your consultant comes in from home promptly and arrives in the emergency department where the patient is at present. It is clear to you that the consultant has been drinking and you can smell alcohol on his breath.

Choose the THREE most appropriate responses from the list below:
A. Let your consultant examine the patient
B. Call your registrar (senior doctor but below consultant) to inform him of the situation
C. Tell the patient you think the consultant is drunk
D. Inform the consultant in front of the patient that you believe you can smell alcohol on his breath
E. Ask the consultant if he feels a colleague should take over from him
F. Seek advice and help of senior emergency department staff regarding the patient's care
G. Allow the consultant to take the patient to theatre
H. Write an e-mail to the GMC, stating that you are concerned that the consultant has a drinking problem

Subject matter expert decided answer key: BEF 